\title{
Hyaluronan and Proteoglycan Link Protein 1 (HAPLN1) Promotes Viability of Rheumatoid Arthritis Fibroblast-Like Synoviocytes
}

\section{Yong Chen}

Shenzhen People's Hospital https://orcid.org/0000-0001-5538-5081

\section{Baojiang Wang}

Maternity and Children Hospital

\section{Yanjuan Chen}

Jinan University

Qunyan Wu

Maternity and Children Hospital

\section{Kutty Selva Nandakumar}

Southern Medical University

Dongzhou Liu ( $\sim$ liu_dz2001@sina.com )

Shenzhen People's Hospital

\section{Research Article}

Keywords: Rheumatoid arthritis, Hyaluronan and proteoglycan link protein 1, fibroblast-like synoviocytes, cell viability, pathogenesis

Posted Date: November 29th, 2021

DOI: https://doi.org/10.21203/rs.3.rs-1088453/v1

License: (c) (i) This work is licensed under a Creative Commons Attribution 4.0 International License.

Read Full License 


\section{Abstract \\ Background}

To investigate HAPLN1 contribution to the viability of RA-FLSs and identify its potential role in RA pathogenesis.

\section{Methods}

Plasma levels and synovial expression of HAPLN1 were compared between healthy controls, and osteoarthritis (OA) and RA patients. Proliferation and migration of RA-FLSs transfected with siHAPLN1, HAPLN1 ${ }^{\text {OE }}$ (over-expression vector) and respective controls or treated with rHAPLN1 were measured by MTT and CCK8 assays as well as wound healing and transwell assays. RT-qPCR and automated WB analysis were used to compare the expression of AMPK- $\triangle$, TNF- $囚$, TGF- $\beta$, ACAN, MMPs, Cyclin-D1 and Ki67 after siHAPLN1 or HAPLN1 ${ }^{\text {OE }}$ transfection. Proteomics and mRNA-seq analysis was done to study the differentially expressed proteins/genes after siHAPLN1 or rHAPLN1 treatment.

\section{Results}

Expression of HAPLN1 was increased in the plasma samples and synovium tissues of RA patients. HAPLN $1{ }^{\text {OE }}$ transfected or rHAPLN1 treated RA-FLSs showed an increased proliferation capacity. However, Si-HAPLN1 has failed to affect the viability of RA-FLSs, though it decreased the migration ability of these cells. On the other hand, HAPLN1 ${ }^{\mathrm{OE}}$ or rHAPLN1 had inhibitory effect on migration. Both si-HAPLN1 and HAPLN $1{ }^{\mathrm{OE}}$ treated RA-FLSs had down-regulated AMPK- $\triangle$ gene expression, while protein level was found to be up-regulated. Furthermore, si-HAPLN1 has down-regulated TNF-囚, MMPs, IL-6, TGF- $\beta$, fibronectin and

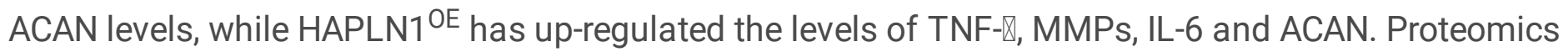
and mRNA-Seq analysis demonstrated HAPLN1 function in the activation of inflammation, proliferation, increased cell adhesion and strengthening of ECM function.

\section{Conclusons:}

HAPLN1 accelerated the proliferation of RA-FLSs but inhibited its migration ability. HAPLN1 network was found to be mainly involved in the activation of inflammation, cell proliferation and increased cell adhesion.

\section{Introduction}

Rheumatoid arthritis (RA) is a chronic autoimmune disease in which joint and cartilage are primarily targeted and destroyed. Pannus formation is one of the main pathological manifestations of the 
disease ${ }^{1}$. During RA development, fibroblast-like synoviocytes (FLSs) produce several pathogenic mediators like cytokines and proteases, which contribute to the destruction of extracellular matrix (ECM) 2,3 . Therefore, FLSs are considered to be one of the major effector cells in RA.

Hyaluronan and proteoglycan link protein 1 (HAPLN1), also known as cartilage link protein, was originally identified from the proteoglycan component extracted from bovine articular cartilage ${ }^{4}$. Physiologically, HAPLN1 maintains the stable aggregation and binding activity of two important ECM macromolecules, hyaluronan acid ( $\mathrm{HA}$ ) and proteoglycan, which contribute to a stable macromolecular structure, and the compression resistance of joints ${ }^{5}$. Indispensable functions of HAPLN1 in the growth of bone, cartilage and heart were well documented ${ }^{5}$. An emerging role for HAPLN1 in oncology was reported recently $5,9,10$. Originally, the most classic disease reported to be associated with HAPLN1 is juvenile rheumatoid arthritis $5,9,10$. Whereas, studies on HAPLN1 function in rheumatic diseases including RA are very few, and the role of the target genes is also unclear. Mainly through genomics research, HAPLN1 relation with spinal degeneration ${ }^{12,13}$, osteoarthritis ${ }^{12,13}$, ankylosing spondylitis ${ }^{15}$ and $\mathrm{RA}^{16}$ was identified.

Interestingly, HAPLN1 showed a significant correlation with disease activity in RA ${ }^{16}$ and found to be one of the most significantly up-regulated genes in RA-FLSs compared to osteoarthritis (OA) FLSs ${ }^{17}$. Based on these observations, it can be inferred that HAPLN1 participates in the development of RA, though its contribution is still unclear.

In cancer-related research, HAPLN1 was shown to have the properties of a oncogene that contributes to an increased susceptibility to lung cancer ${ }^{18}$, aggressiveness of hepatocellular carcinomas ${ }^{19}$, and drug resistance to multiple myeloma ${ }^{20}$. Therefore, we hypothesized that HAPLN1 may increase the viability of RA-FLSs, mimicking the behavior of cancer cells, which may lead to an aggressive pannus formation and joint damage ${ }^{21}$.

\section{Material And Methods}

\section{Patients' and Samples}

We enrolled 12 age and gender matched medical staff as healthy controls. Their blood samples as well as 61 RA and 20 OA patients' samples were used for measuring HAPLN1 levels by ELISA. Synovium samples were collected by arthroscopic surgery done with 20 RA and 17 OA patients and used for IHC staining of HAPLN1. The inclusion and exclusion criteria and general information such as age, gender, disease activity and disease course were reported earlier ${ }^{21}$ and summarized in supplementary table 1 and 2 .

\section{Enzyme linked immunosorbent assay (ELISA)}

Blood samples of HC, OA and RA patients were centrifuged after standing at room temperature for $2 \mathrm{~h}$ at 1,500 $\mathrm{g}$ for 10 min to collect the plasma and HAPLN1 levels were detected by ELISA (RayBiotech, USA). Plasma AMPK levels in RA patients were also evaluated by ELISA according to the manufacturer's 
protocol (Jianglaibio, China). The SuPerMax 3000FA absorbance microplate reader (Flash Co. Ltd., China) was used to read the optical density (OD) value at $450 \mathrm{~nm}$ and concentrations of specific proteins were calculated based on the standard curve.

\section{Immunohistochemical (IHC) staining for HAPLN1}

Synovium samples collected from 20 RA and 17 OA patients for IHC staining of HAPLN1 were prepared as reported earlier ${ }^{17}$. Rabbit monoclonal anti-HAPLN1 antibody (Abcam, USA) was added as primary antibody $(1: 50)$ and incubated for $2 \mathrm{~h}$ at $37^{\circ} \mathrm{C}$. Biotin-conjugated goat anti-rabbit antibodies (ZSGB-Bio, China), streptavidin-peroxidase conjugate, and diaminobenzidine were used as the detecting system. IHC stained sections were semi-quantified under a microscope. The staining intensity was counted as none ( 0 points), weakly positive $(1+)$, moderately positive $(2+)$ and strong positive $(3+)$, and the percentage of positive cells was obtained to calculate the H-Score, using the formula H-Score $=(\%$ at 0$) \times 0+(\%$ at $1+) \times$ $1+(\%$ at $2+) \times 2+(\%$ at $3+) \times 3$. The range of $\mathrm{H}$ score for each slice was between $0-300^{22}$.

\section{Isolation and culture of RA-FLSs}

Primary RA-FLSs were acquired from 3 untreated RA patients having appropriate diagnostic criteria. Isolation and culture of RA-FLSs were reported as before ${ }^{17}$. Briefly, FLSs were isolated by enzyme digestion and subsequently cultured in Dulbecco's modified essential medium (DMEM) containing 10\% fetal bovine serum (FBS, Invitrogen) and antibiotics (penicillin and streptomycin) at $37^{\circ} \mathrm{C}$ with $5 \% \mathrm{CO}_{2}$. Cells cultured between passages 4 and 9 were used in this study.

\section{Small Interfering RNA (siRNA) HAPLN1 preparation and transfection}

RA-FLSs at 60 - 70\% confluency were transfected with siRNAs (Ribobio Company, China) at $50 \mathrm{nM}$ with Lipofectamine $^{\mathrm{TM}} 3000$ reagent (Invitrogen, USA). The following siRNA sequences were used: control siRNA (confidential sequence information) and 3 siRNAs of HAPLN1, si-1 (5'-AGGGTAGAGTGTTTCTGAA-3'), si-2 (5'-CCTGGAAAATTCTCGGATA) and si-3 (5'-ACCTCACTCTGGAAGATTA-3'). All the three siRNAs effectively silenced HAPLN1 expression (supplementary figure 2A), and si-1 was selected randomly and used in subsequent studies.

\section{HAPLN1 over-expression vector preparation and transfection}

For HAPLN1 ${ }^{\text {OE }}$ RA-FLSs experiments, HAPLN1 over-expression plasmid and its control were constructed and packaged by Ubigene Biosciences (Guangzhou, China). The stbl3 strain plasmid cytomegalovirus vector infected cells were cultured in LB medium (QDRS biotec, China) with $100 \mu \mathrm{g} / \mathrm{ml}$ of ampicillin under $37^{\circ} \mathrm{C}, 225 \mathrm{rpm}$ for $24 \mathrm{~h}$. The HAPLN1 ${ }^{\mathrm{OE}}$ plasmid vector and its control were then isolated with Genopure PIsmid Maxi Kit (Roche, USA). RA-FLS at $60-70 \%$ confluency were transfected with HAPLN1 ${ }^{\text {OE }}$ vector or its negative control with Lipofectamine ${ }^{T M} 3000$ reagent (Invitrogen). The effects of HAPLN1 ${ }^{\text {OE }}$ plasmid vector were shown in the supplementary figure $2 \mathrm{~B}$. 


\section{MTT assay}

MTT assay was used to ascertain FLSs viability transfected with si-HAPLN1, HAPLN1 ${ }^{\text {OE }}$ or their corespondent controls, or treated with rHAPLN1 (Recombinant human HAPLN1 protein, Abcam, USA) at different concentrations $(0,25$ and $50 \mathrm{ng} / \mathrm{ml}$ ). FLSs samples (si-HAPLN1 vs its negative control, HAPLN1 ${ }^{\mathrm{OE}}$ vs its negative control, or treated with different concentrations of rHAPLN1) digested using $0.25 \%$ pancreatin were transferred to 96 -well plates with 3-5 $\times 10^{3}$ cells/well. At different time points (24, 48 and $72 \mathrm{~h}$ ), proliferation viability was measured using MTT assay kit (Abcam).

\section{CCK-8 assay}

Cell viability after transfection with si-HAPLN1, HAPLN1 ${ }^{\mathrm{OE}}$ or their respective controls, or treated with different concentrations of rHAPLN1 was also determined using Cell Counting Kit-8 (CCK-8, Molecular Technology, Japan) assay.

\section{TUNEL assay}

FLSs transfected with si-HAPLN1, HAPLN1 ${ }^{\text {OE }}$ or their corespondent controls, or treated with rHAPLN1 (0 or $50 \mathrm{ng} / \mathrm{ml}$ ) were digested and transferred to 6-well plates with 2-3 $\times 10^{5}$ cells/well, cultured for $48 \mathrm{~h}$, and stained by One Step TUNEL Apoptosis Assay Kit (Beyotime, China). Apoptosis rate was calculated under fluorescence microscope (Leica, Germany) with the excitation wavelength at $550 \mathrm{~nm}$ (Cy3), and the emission wavelength at $570 \mathrm{~nm}$ (red fluorescence).

\section{Flow cytometry for FLSs apoptosis}

FLSs apoptosis treated with rHAPLN1 was measured using Annexin V-FITC/PI Cell Apoptosis Detection Kit (Vazyme, China) by flow cytometry. After FLSs were treated with rHAPLN1 (0 or $50 \mathrm{ng} / \mathrm{ml}$ ) for $48 \mathrm{~h}$ in 6-well plates, the cells were collected $\left(3 \times 10^{5} /\right.$ well), washed twice with PBS, re-suspended in $500 \mu \mathrm{l}$ X binding buffer, mixed with Annexin-V-fluorescein isothiocyanate ( $\mathrm{FITC}, 5 \mu \mathrm{l})$ and propidium iodide ( $\mathrm{PI}, 5$ $\mu \mathrm{l})$, and analyzed using a flow cytometer (BD FACSCanto ${ }^{\mathrm{TM}} \mathbb{\otimes}, \mathrm{USA}$ ). The scatter diagram was distributed as follows: Q3, healthy cells (FITC-/PI-); Q2, apoptotic cells at an advanced stage (FITC+/PI+); Q4, apoptotic cells at an early stage (FITC+/PI-). Apoptosis rate was calculated as ratio of apoptotic cells in $\mathrm{P} 2(\mathrm{Q} 4+\mathrm{Q} 2)$.

\section{Wound healing assay}

Wound healing assay was done to evaluate the migration viability of FLSs transfected either with siHAPLN1, HAPLN1 ${ }^{\mathrm{OE}}$ or their corespondent controls, or treated with different concentrations $(0,25$ and 50 $\mathrm{ng} / \mathrm{ml}$ ) of rHAPLN1. FLSs samples (si-HAPLN1 vs its negative control, HAPLN1 ${ }^{\mathrm{OE}}$ vs its negative control, or treated with different concentrations of rHAPLN1) were transferred to 6-well plates with $3 \times 10^{5}$ cells/well and cultured with serum free - RPMI 1640 medium. At different time points, migration viability was measured by wound healing assay as previously reported ${ }^{17}$.

\section{Transwell assay}


Transwell assay was also performed to evaluate the migration capacity of FLSs transfected with siHAPLN1, HAPLN1 ${ }^{\text {OE }}$ or their respective controls, or treated with rHAPLN1. FLSs in each set of experiment were re-suspended after culturing for $24 \mathrm{~h}$. Transwell assay procedure was described earlier ${ }^{17}$.

\section{Quantitative real-time polymerase chain reaction (qPCR)}

Total RNA from FLSs transfected with si-HAPLN1, HAPLN1 ${ }^{\mathrm{OE}}$ or their respective controls, or treated with rHAPLN1 was prepared using TRIzoI® Reagent (Thermo Scientific, USA) and quantified using Qubit (Thermofisher, USA). RNA was reverse transcribed into cDNA using PrimeScript ${ }^{T M}$ RT Master Mix (Takara, Japan). The reaction mixture contained $5 \mu$ of $2 \times$ TB Green Premix Ex Taq II (Takara, Japan), $3 \mu$ of nuclease-free water, $1 \mu \mathrm{l}$ of CDNA, $0.4 \mu \mathrm{l}$ of each gene-specific primer and $0.2 \mu \mathrm{l}$ of ROX reference dye. The qRT-PCR analysis was performed using Applied Biosystems ViiA ${ }^{\text {TM }} 7$ Real-Time PCR System

(Thermofisher, USA). Each value represents an average from three independent biological replicates.

GAPDH gene expression was used for data standardization. The fold change was calculated using $2^{-\triangle \triangle C t}$ method. Primers of GAPDH, AMPK- $\varangle$, TNF- $\bigotimes$, IL-6, TGF- $\beta$, ACAN, fibronectin, collagen II, MMP1, MMP3, MMP9, cyclin-D1 and Ki-67 are given in the supplementary Table 3.

\section{Automated western blot analysis}

Total proteins from FLSs transfected with si-HAPLN1, HAPLN1 ${ }^{\mathrm{OE}}$ or their respective controls for $48 \mathrm{~h}$ were extracted with Cell Lysis Buffer (Cell Signaling, USA) and their concentration was measured using a BCA Protein Assay Kit (Merck, USA). Relative changes in HAPLN1, pAMPK-a, IL-6, TNF-a, MMP1, MMP3 and MMP9 protein levels were determined. Expression of $\beta$-tublin was selected as internal reference. Capillary electrophoresis and western blot analysis were carried out using reagents provided in the kit and following instructions in the user manual (ProteinSimple WES, USA) as previously reported ${ }^{17}$. Rabbit antiHAPLN1 antibody (Abcam, USA), Rabbit anti-TNF-a, pAMPK-a, MMP-1, MMP-3, IL-6, and $\beta$-tublin specific mAbs (Cell Signaling, USA) were used (1:100). Goat anti-rabbit secondary antibodies were provided in the ProteinSimple WES kit and applied as instructed. Data were analyzed using an in-built Compass software SW 4.0. The truncated and full-length target protein intensities (area under the curve) were normalized to that of tubulin peak (control). In most of the figures, electropherograms are represented as pseudo-blots, generated using Compass software.

\section{Proteomics study}

Label-free proteomics study was applied to FLSs transfected by si-HAPLN1, or treated by rHAPLN1 (50 $\mathrm{ng} / \mathrm{ml}$ ) and their controls for 48 hours (for management of each group is seen in table 4) by PTMBiolabs, Inc. (Hangzhou, China). Each concentration with 3 biological replicates. Cell samples processed as reported $^{23}$. LC-MS/MS proteomics analysis was performed on an EASY-nLC 1000 ultra-performance liquid chromatography (UPLC) system, followed by MS/MS using Q Exactive Plus (ThermoFisher Scientific, USA) coupled online to the UPLC system. The MS/MS data were retrieved by the Maxquant search engine (v1.6.6.0). A human database was searched (Swiss-Prot). The decoy database antilibrary was used to the reduce false positive rate (FDR). The FDR was adjusted to $<1 \%$, and the minimum score 
for modified peptides was set $>40$. Proteins with a fold-change $\geq 1.50$ or $\leq 0.67$ between si-HAPLN1, rHAPLN1 and their controls were considered as expression significant. Based on the protein sequence alignment method, the protein domain functions were defined by InterProScan (http://www.ebi.ac.uk/interpro/). Functional annotation enrichment of DEGs were performed by Gene Ontology (GO) annotation analysis and KEGG analysis. The enrichment significant was identified as $p<$ 0.05 in Fisher's exact test and $\mathrm{q}<0.05$ in Benjamini-Hochberg's procedure.

\section{High-throughput mRNA sequencing}

High-throughput RNA sequencing was performed using FLSs after treatment with rHAPLN1 (0 and 50 $\mathrm{ng} / \mathrm{ml}$ ) for $48 \mathrm{~h}$. Each concentration was tested thrice. RNA-seq and high throughput sequencing were conducted by Seqhealth Technology Co., Ltd (Wuhan, China). Total RNA $(2 \mu \mathrm{g})$ was used for stranded RNA sequencing library preparation using KCTM Stranded mRNA Library Prep Kit for Illumina ${ }^{\circledR}$ (Seqhealth Co., Ltd. China). PCR products corresponding to 200-500 bps were enriched, quantified and sequenced with Novaseq 6000 sequencer (Illumina), PE150 model. Raw sequencing data was first filtered by Trimmomatic (v. 0.36), and low-quality reads were discarded. The reads contaminated with adaptor sequences were trimmed. Clean data were mapped to the human reference genome from UCSC (https://genome.ucsc.edu/) using STRA software (v. 2.5.3a) with default parameters. Reads mapped to the exon regions of each gene were counted by feature Counts (Subread-1.5.1; Bioconductor) and then RPKMs were calculated. DEGs between groups were identified using the edgeR package (v. 3.12.1) in $R$ studio software (version 3.6). A p-value cutoff of 0.05 and fold-change cutoff of 2.0 were used to judge the statistical significance of gene expression differences. The volcano plot was drawn with the ggplot2 package in R studio. Heatmaps of pathway enrichment analysis of DEGs were generated using Metascape (http://metascape.org) ${ }^{24}$ and $p$ value less than 0.05 was considered to be statistically significant. Gene ontology (GO) analysis and Kyoto encyclopedia of genes and genomes (KEGG) enrichment analysis for DEGs were done uisng KOBAS software (v. 2.1.1) with a p value cutoff of 0.05 . To compare transcriptome characteristics of rHAPLN1 and PBS groups, GSEA software (version 4.0.0) was used. Annotated pathway files (c5.go.bp.v7.4.symbols.gmt) were downloaded in the MSigDB database (http://www.gsea-msigdb.org/gsea/msigdb/collections.jsp). Pathways with P value less than 0.05 and false discovery rate (FDR) less than 0.2 were considered to be significantly enriched.

\section{Statistical analysis}

Statistical analysis was performed using GraphPad Prism 9.0 software. All the data were given as mean \pm SD. Differences between two groups were evaluated for statistical significance using Student's t-test. One-way ANOVA with Tukey's multiple comparisons test was used to evaluate the differences between three or more groups. Pearson correlation coefficient was calculated using "cor" function in R studio. Correlations were evaluated using liner regression and correlation tests. $p<0.05$ was considered as statistically significant.

\section{Results}




\section{Increased HAPLN1 expression in synovium and plasma of RA patients}

Our mRNA sequencing analysis showed HAPLN1 expression in RA FLSs was higher than OA FLSs ${ }^{17}$ (Figure 1A). Here, we report HAPLN1 expression in the synovium was significantly higher in RA than OA patients (Figure 1B, participants' information is given in the supplementary table 1). Similarly, HAPLN1 levels in the plasma of RA patients was also higher than OA patients and healthy controls (Figure 1C, participants' information is given in the supplementary table 2). In addition, plasma HAPLN1 levels have positively correlated with the severity of disease in RA patients $(r=-0.311, p=0.038)$ (supplementary figure 1A). Interestingly, patients with less severe disease course had higher HAPLN1 levels (supplementary figure 1B). However, no correlation between HAPLN1 level with other indexes of disease activity like ESR and CRP was observed, but it positively correlated with plasma AMPK levels $(r=0.693, p$ $<0.0001$ ) (supplementary figure 1C \& D).

\section{HAPLN1 increased the proliferation but inhibited mobility of RA-FLSs}

All the three siRNAs prepared have effectively down-regulated HAPLN1 mRNA expression, while transferring HAPLN1 over-expression vector into RA-FLSs up-regulated its expression (Supplementary figure 2). Using MTT and CCK8 assays, we have shown that si-HAPLN1 transfection has failed to affect the proliferation of RA-FLSs (Figure 2A and supplementary figure 3). However, it has significantly increased the apoptotic ratio of RA-FLSs as observed with TUNEL assay (Figure 2B). Both wound healing and transwell assays showed an increased migration ability of RA-FLSs after si-HAPLN1 transfection (Figure 2C \& D), which could be reversed by adding r-HAPLN1 $(50 \mathrm{ng} / \mathrm{ml})$ to the culture medium (Figure 2D).

Conversely, after transfection with HAPLN1 over-expression vector, RA-FLSs showed an increased proliferation and a decreased apoptotic ratio (Figure $3 A$ \& B and supplementary figure 4). Wound healing assay suggested a decreased level of migration with HAPLN1 ${ }^{\mathrm{OE}}$ transfected RA-FLSs (Figure 3C). Whereas, rHAPLN1 confirmed the effects on RA-FLSs, which significantly enhanced the proliferation activity of RA-FLSs (Figure 4A, and supplementary figure 5) and reduced RA-FLSs apoptosis, especially during early phase (Figure 4B \& C). The wound healing and transwell assays demonstrated that rHAPLN1 inhibited the migration ability of RA-FLSs (Figure 4D \& E).

\section{Expression of HAPLN1 affects RA-FLSs secreted cytokines and molecules}

The si-HAPLN1 transfection into RA-FLSs inhibited the expression of AMPK- $\varangle$, TNF- $\nabla$, IL-6, TGF- $\beta$, ACAN, fibronectin, collagen II, MMP1, MMP3, MMP9, cyclin-D1 and Ki-67 (Figure 5A \& B). Automated WB assay confirmed successful silencing of HAPLN1. However, at the protein level unlike mRNA expression, the

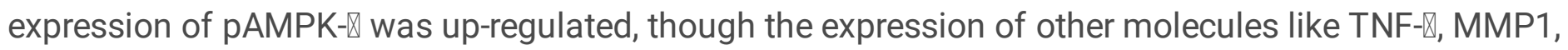


MMP3, and MMP9 were inhibited similar to mRNA expression (Figure 5C). HAPLN1 over-expression in RAFLSs has up-regulated the expression of TNF-囚, IL-6, ACAN, MMP9 and Ki-67, while AMPK-囚and cyclin-D1 expressions were down-regulated (Figure 5D). WB analysis confirmed the successful transfection of

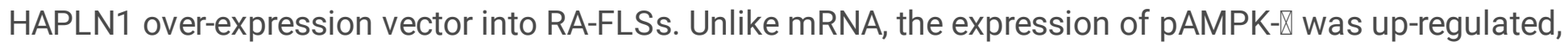
while TNF-囚, IL-6, MMP1, MMP3 showed an up-regulation trend (Figure 5E).

In order to better understand potential interactions between these molecules, we collected multiple expression data of relative mRNA levels from NC groups and applied Pearson correlation coefficient to identify any potential correlations. Importantly, HAPLN1 level has positively correlated with AMPK-区, which is in accordance with its plasma levels, and also with our previous findings using RA-FLSs ${ }^{17}$. In addition, HAPLN1 levels have positively correlated with inflammatory molecules like MMP1, MMP9, TNFQ, and IL-6. Furthermore, HAPLN1 levels showed positive correlations with ECM modulators like TGF-ßand Collagen type II, while a negative correlation was observed with the inflammatory molecules MMP1, MMP-3, MMP9, TNF- $\mathrm{X}$, and IL-6 (supplementary figure 6).

\section{Proteome and mRNA-seq analysis of HAPLN1 functions in RA-FLSs}

In order to understand HAPLN1 functions in RA-FLSs, proteomic analysis of si-HAPLN1 and rHAPLN1 treated RA-FLSs, and transcriptome analysis of rHAPLN1 treated RA-FLSs were done. In the proteomic study, RA-FLSs from NC, si-HAPLN1, and rHAPLN1 groups included 443,973 matched spectra in total, and 4,184 quantifiable proteins were identified (Table 1). Principal component analysis (PCA) plot indicated a high aggregation between duplicated samples, demonstrating the quantitative repeatability of experiments (supplementary figure 7). Among the identified proteins, 14 were up-regulated and 47 were down-regulated after si-HAPLN1 transfection. Besides, 101 proteins were up-regulated, and 82 were downregulated by rHAPLN1 treatment compared to NC (Figure 6A).

Table 1

Overview of protein identification

\begin{tabular}{|ll|}
\hline Title & Number \\
\hline Total number of spectra & 1022232 \\
\hline Number of matched spectra & 443973 \\
\hline Peptides & 38576 \\
\hline Unique peptides & 37344 \\
\hline Identified proteins & 4944 \\
\hline Quantifiable proteins & 4184 \\
\hline
\end{tabular}

Note: 1. Total number of spectra includes number of total spectra and number of secondary spectra generated by mass detection; 2 . Number of matched spectra includes the number of effective spectra and 
the number of spectra matching the theoretical secondary spectra; 3 . Peptides include the number of identified peptides and the number of peptide sequences resolved by the matching result; 4 . Unique peptides include the number of unique identified peptides and, the number of unique peptide sequences resolved by the matching result; 5 . Identified proteins include the number of identified proteins; 6 . Quantifiable proteins include quantitative protein numbers and the number of proteins quantified by specific peptides.

KEGG enrichment analysis showed up-regulated DEGs in rHAPLN1 treated RA-FLSs were enriched in pathways including protein digestion, ECM-receptor interaction, focal adhesion, PI3K-Akt signaling pathway, JAK-STAT signaling pathway, cholesterol metabolism and metabolism of xenobiotics by cytochrome P450. On the other hand, down-regulated DEGs were enriched in pathways of cellular senescence, glutathione metabolism, rheumatoid arthritis and cytokine-cytokine receptor interaction (Figure 6C and supplementary figure 8). The down regulated DEGs in si-HAPLN1 transfected RA-FLSs were enriched in pathways of coronavirus disease (COVID-19), ribosome, systemic lupus erythematosus and TNF signaling pathways, while the up-regulated DEGs failed to be enriched in any of the known pathways (Figure 6C and supplementary figure 9).

Next, mRNA sequencing analysis was done with rHAPLN1 or PBS treated RA-FLSs. Principal component analysis (PCA) plot showed a high aggregation between duplicated samples, suggesting the quantitative repeatability of experiments (supplementary figure 10). Among the 504 DEGs detected, 439 were upregulated while 65 were down-regulated (Figure 7A). However, only 2 DEGs (up-regulated LRP1 and downregulated CRIP1) were in accordance with the results found in proteome analysis (Figure 7A \& B).

Metascape pathway analysis showed the up-regulated DEGs treated by rHAPLN1 were mainly enriched in GTPase Cycle, cell cycle and regulation of cell division (Figure 7C), which were confirmed by proliferation and apoptosis studies. Moreover, GSEA analysis were performed to compare rHAPLN1 and PBS groups. The results showed that rHAPLN1 group has positively associated with the pathways of extracellular matrix structural constituents, alpha actin binding, metalloaminopeptidase activity, proteoglycan binding, focal adhesion, regulation of protein exit from endoplasmic reticulum, lipid translocation, regulation of androgen receptor signaling pathway, retrograde axonal transport, dendritic spine development, peptide cross linking and insulin like growth factor receptor signaling pathway (Supplementary figure 11).

\section{Discussion}

Here, we confirmed an increased HAPLN1 expression in the synovium and plasma samples from RA patients. Over-expression of HAPLN1 or treatment with rHAPLN1 increased the proliferation but decreased apoptosis of RA-FLSs. However, si-HAPLN1 transfection has failed to show any effect on proliferation, though it induced significant apoptosis of RA-FLSs. HAPLN1 was discovered 50 years ago, with a wide range of physiological effects as documented. HAPLN1 interacts with the globular domains of hyaluronic acid and proteoglycans, such as aggrecan, versican anda-trypsin inhibitor in various 
extracellular matrices to form a stable ternary complex ${ }^{25}$ and contributes to the compression resistance and shock absorption of the joints. Thus, HAPLN1 plays an important role in cartilage formation and homeostasis. HAPLN1-deficient mice showed a series of cardiac malformations, including atrial septal and myocardial defects with a significant reduction in the level of multifunctional proteoglycans ${ }^{8}$. In addition, HAPLN1 also plays an important role in regulating the development of the central nervous system $^{26}$. In the embryonic development of zebrafish, the expression of HAPLN1 was observed in the paraxial cells at the budding stage. During the somatogenesis stage, HAPLN1 was observed in the condyles, basal plate, inferior notochord and rhombohedral protonode branchial arch ${ }^{27}$. Whereas, during the process of chondrogenesis and differentiation of human mesenchymal stem cells (hMSCs) in vitro, HAPLN1 reached its peak level of expression at 6-12 days ${ }^{28}$. Perinatal mice with inactivated HAPLN1 developed lethal achondroplasia and the extremities and vertebral cartilage lacked proteoglycan deposition with a reduced number of hypertrophic chondrocytes ${ }^{29}$.

HAPLN1 was found to be one of the most obviously up-regulated DEGs in RA-FLSs and the activation of AMP-activated protein kinase (AMPK) by metformin in RA-FLSs increased the secretion of HAPLN1 ${ }^{17}$. Here, a significant positive correlation between plasma AMPK and HAPLN1 levels was identified. Considering AMPK mediated inhibition of a broad range of inflammatory reactions involving multiple biological pathways, it is plausible that an increased HAPLN1 might be the way, how metformin benefits RA patients ${ }^{17}$, and also how body reacts to inflammation induced stress responses. However, HAPLN1 ${ }^{\text {OE }}$ or rHAPLN1 treated RA-FLSs showed an increased proliferation, which is contradictory to our previous assumption about HAPLN1 as a protective factor. On the other hand, si-HAPLN1 decreased the migration capability while HAPLN1 ${ }^{\text {OE }}$ or rHAPLN1 treated RA-FLSs showed an inhibitory effect. At this point, HAPLN1 seemed to be a protective molecule. Thus, it seems there is a dilemma in clarifying the role of HAPLN1 in RA-FLSs viability by functional studies. It's interesting to note that both results could possibly be consistent with the findings in cancer studies, because an increased HAPLN1 seems associated with more aggressiveness, and leading to stemness of various cancers ${ }^{18,19,30}$, while achieving robust ECM to restrict metastasis of cancer cells ${ }^{9,10}$.

Earlier interactions between HAPLN1 and other molecules beyond hyaluronic acid and proteoglycans

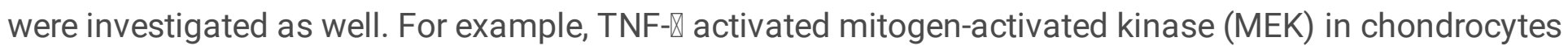
regulates the expression of HAPLN1, and controls the catabolism and anabolism of the extracellular matrix of chondrocytes ${ }^{31}$. In multiple myeloma cells, HAPLN1 can activate the NF-『B pathway to acquire resistance to bortezomib ${ }^{20}$. In granulosa cells, HAPLN1 was proposed to be promoted through PKARUNX1/RUNX2 pathway ${ }^{32}$, which was earlier confirmed in metformin treated RA-FLSs ${ }^{17}$. In this study, relative mRNA expression of AMPK- $\$ and HAPLN1 in untreated RA-FLSs showed a positive correlation. However, both si-HAPLN1 and HAPLN1 ${ }^{\mathrm{OE}}$ treated RA-FLSs had down-regulated AMPK- $\$ expression at mRNA but not protein level, which suggests a complex feedback circle between AMPK- $\$ and HAPLN1 . Furthermore, on silencing HAPLN1, pro-inflammatory factors such as TNF-X, MMPs and IL-6 as well as structure related molecules like TGF- $\beta$, Fibronectin and ACAN were down-regulated. Conversely, 


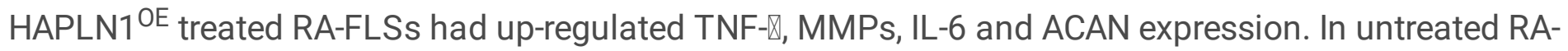
FLSs, relative mRNA expression of HAPLN1 was positively associated with TGF- $\beta$ and IL-6. The Ki-67 has been widely used as a proliferation marker for most human tumor cells for decades ${ }^{33}$, which was decreased after si-HAPLN1 transfection but increased by HAPLN1 ${ }^{\mathrm{OE}}$ treatment. In addition, cyclin D1 has an important part in regulating cell proliferation during $\mathrm{G} 1$ phase of the cell cycle. Given the frequent overexpression of cyclin D1 in cancer cells, its expression appears to be closely linked with carcinogenesis ${ }^{34}$. The oncological findings suggest that cyclin D1 has a central role in mediating invasion and metastasis of cancer cells by controlling Rho/ROCK signaling and matrix deposition of thrombospondin- ${ }^{35}$. In this study, the mRNA expression levels of cyclin D1 in RA-FLSs was significantly decreased by HAPLN1 ${ }^{\mathrm{OE}}$ treatment, which might possibly explain its inhibitory ability on FLSs migration.

Proteomic and mRNA-seq results showed HAPLN1 function in RA-FLSs from a holistic view. Proteomic analysis suggested si-HAPLN1 transfected RA-FLSs were enriched in pro-inflammatory pathways with down-regulated DEGs. It is not strange that mRNA and protein levels seem to have a low correlation, as the multi-step process of gene expression involves transcription, translocation, turnover of mRNAs and proteins $^{36}$. Although only 2 DEGs overlapped with proteomic and transcriptional studies with rHAPLN1 treated RA-FLSs, the omics study reflected activation of inflammation, proliferation, an increased cell adhesion and strengthening of ECM function.

\section{Conclusion}

In conclusion, HAPLN1 accelerates proliferation of RA-FLSs to form a pathological pannus, mimicking cancer cells. Based on physiological development and oncology studies, HAPLN1 seems to be an oncogene, however, with an opposing function on cell adherence and inhibition of migration ability. These findings were also confirmed by molecular network consisting of MMPs, IL-6, Ki-67 and TGF- $\beta$. Thus, HAPLN1 can be considered as a pathogenic factor; however, more questions are inevitable: Why HAPLN1 expression is higher in RA patients with shorter disease course? HAPLN1 seems closely related with metabolism, and positively correlated with AMPK, but which one is an upstream regulator? What would happen if HAPLN1 is silenced in vivo? Is there any potential benefits possible with HAPLN1 with its ossification, joint protection or inhibitory effect on FLSs migration under certain circumstances? This primary study is the first-time report on HAPLN1 effect on the viability of RA-FLSs, and more experiments are needed for clear deciphering of its role in RA.

\section{Abbreviations}

HAPLN1: Hyaluronan and proteoglycan link protein 1

RA: rheumatoid arthritis

OA: Osteoarthritis 
ECM: extracellular matrix

FLSs: fibroblast-like synoviocytes

ELISA: Enzyme linked immunosorbent assay

IHC: Immunohistochemical

siRNA: Small Interfering RNA

qPCR: Quantitative real-time polymerase chain reaction

AMPK: AMP-activated protein kinase

TNF: Tumor necrosis factor

IL: Interleukin

TGF: Transforming growth factor

ACAN: Aggrecan

MMP: Matrix Metallopeptidase

DEG: Differential expressed gene

\section{Declarations}

\section{Ethical Approval and Consent to participate}

All participants were at least 18 years of age and gave written informed consent for this study, which was approved by the Ethics Committee of Integrated Traditional Chinese and Western Medicine Hospital, Southern Medical University, China (approval no. FZXYEC-2017-002) in accordance with the Declaration of Helsinki.

\section{Consent for publication}

Not applicable.

\section{Availability of supporting data}

The datasets used and/or analysed during the current study are available from the corresponding author or first author on reasonable request.

\section{Competing interests}

The authors declare no competing interests. 


\section{Funding}

The study was funded by the National Natural Science Foundation of China (project No. 8197060898), and the National Key Research and Development Program of China (2019YFC0840603).

\section{Authors' contributions}

Concept and design: YC and DZL. Experiment performance: YC, BJW, YQW. Acquisition, analysis and interpretation of data: YC, YJC and KSN. Drafting the manuscript: YC, KSN and BJW.

\section{Acknowledgements}

We thank the patients who have participated in the study, and we are grateful for the time and effort from all the investigators, doctors, study nurses, research assistants at the study centers for their invaluable contribution to this study.

\section{Authors' information}

Not applicable.

\section{References}

1. Lin YJ, Anzaghe M, Schulke S. Update on the pathomechanism, diagnosis, and treatment options for rheumatoid arthritis. Cells-Basel. 2020,9(4) doi: 10.3390/cells9040880

2. Nygaard G, Firestein GS. Restoring synovial homeostasis in rheumatoid arthritis by targeting fibroblast-like synoviocytes. Nat Rev Rheumatol. 2020,16(6):316-333. doi: 10.1038/s41584-0200413-5

3. Ganesan R, Rasool M. Fibroblast-like synoviocytes-dependent effector molecules as a critical mediator for rheumatoid arthritis: Current status and future directions. Int Rev Immunol. 2017,36(1):20-30. doi: 10.1080/08830185.2016.1269175

4. Swann DA, Powell S, Broadhurst J, Sordillo E, Sotman S. The formation of a stable complex between dissociated proteoglycan and hyaluronic acid in the absence of a link protein. Biochem $\mathrm{J}$. 1976,157(2):503-506. doi: 10.1042/bj1570503

5. Evanko SP, Gooden MD, Kang I, Chan CK, Vernon RB, Wight TN. A role for HAPLN1 during phenotypic modulation of human lung fibroblasts in vitro. J Histochem Cytochem. 2020,68(11):797-811. doi: $10.1369 / 0022155420966663$

6. Yao $B$, Zhang M, Liu M, et al. Identification of the miRNA-mRNA regulatory network of antler growth centers. J Biosci. 2019,44(1)

7. Watanabe $\mathrm{H}$, Yamada Y. Chondrodysplasia of gene knockout mice for aggrecan and link protein. Glycoconj J. 2002,19(4-5):269-273. doi: 10.1023/A:1025344332099 
8. Wirrig EE, Snarr BS, Chintalapudi MR, et al. Cartilage link protein 1 (Crtl1), an extracellular matrix component playing an important role in heart development. Dev Biol. 2007,310(2):291-303. doi: 10.1016/j.ydbio.2007.07.041

9. Ecker BL, Kaur A, Douglass SM, et al. Age-Related changes in HAPLN1 increase lymphatic permeability and affect routes of melanoma metastasis. Cancer Discov. 2019,9(1):82-95. doi: 10.1158/2159-8290.CD-18-0168

10. Kaur A, Ecker BL, Douglass SM, et al. Remodeling of the collagen matrix in aging skin promotes melanoma metastasis and affects immune cell motility. Cancer Discov. 2019,9(1):64-81. doi: 10.1158/2159-8290.CD-18-0193

11. Danieli MG, Markovits D, Gabrielli A, et al. Juvenile rheumatoid arthritis patients manifest immune reactivity to the mycobacterial $65-\mathrm{kD}$ a heat shock protein, to its $180-188$ peptide, and to a partially homologous peptide of the proteoglycan link protein. Clin Immunol Immunopathol. 1992,64(2):121128. doi: 10.1016/0090-1229(92)90189-u

12. Urano T, Narusawa $K$, Shiraki M, et al. Single-nucleotide polymorphism in the hyaluronan and proteoglycan link protein 1 (HAPLN1) gene is associated with spinal osteophyte formation and disc degeneration in Japanese women. Eur Spine J. 2011,20(4):572-577. doi: 10.1007/s00586-010-15980

13. Erwin WM, Desouza L, Funabashi M, et al. The biological basis of degenerative disc disease: Proteomic and biomechanical analysis of the canine intervertebral disc. Arthritis Res Ther. 2015,17:240. doi: 10.1186/s13075-015-0733-z

14. Zheng J, Wu C, Ma W, et al. Abnormal expression of chondroitin sulphate $\mathrm{N}$ acetylgalactosaminyltransferase 1 and Hapln-1 in cartilage with Kashin-Beck disease and primary osteoarthritis. Int Orthop. 2013,37(10):2051-2059. doi: 10.1007/s00264-013-1937-y

15. Layh-Schmitt G, Lu S, Navid F, et al. Generation and differentiation of induced pluripotent stem cells reveal ankylosing spondylitis risk gene expression in bone progenitors. Clin Rheumatol. 2017,36(1):143-154. doi: 10.1007/s10067-016-3469-5

16. Galligan CL, Baig E, Bykerk V, Keystone EC, Fish EN. Distinctive gene expression signatures in rheumatoid arthritis synovial tissue fibroblast cells: Correlates with disease activity. Genes Immun. 2007,8(6):480-491. doi: 10.1038/sj.gene.6364400

17. Chen Y, Qiu F, Yu B, et al. Metformin, an AMPK activator, inhibits activation of FLSs but promotes HAPLN1 secretion. Mol Ther Methods Clin Dev. 2020,17:1202-1214. doi:

10.1016/j.omtm.2020.05.008

18. Jones $\mathrm{CC}$, Bradford $\mathrm{Y}, \mathrm{Amos} \mathrm{Cl}$, et al. Cross-Cancer pleiotropic associations with lung cancer risk in african americans. Cancer Epidemiol Biomarkers Prev. 2019,28(4):715-723. doi: 10.1158/10559965.EPI-18-0935

19. Mebarki S, Désert R, Sulpice L, et al. De novo HAPLN1 expression hallmarks Wnt-induced stem cell and fibrogenic networks leading to aggressive human hepatocellular carcinomas. Oncotarget. 2016,7(26):39026-39043. 
20. Huynh M, Pak C, Markovina S, et al. Hyaluronan and proteoglycan link protein 1 (HAPLN1) activates bortezomib-resistant NF-kappaB activity and increases drug resistance in multiple myeloma. J Biol Chem. 2018,293(7):2452-2465. doi: 10.1074/jbc.RA117.000667

21. Bustamante MF, Garcia-Carbonell R, Whisenant KD, Guma M. Fibroblast-like synoviocyte metabolism in the pathogenesis of rheumatoid arthritis. Arthritis Res Ther. 2017,19(1):110. doi: 10.1186/s13075017-1303-3

22. Pierceall WE, Wolfe M, Suschak J, et al. Strategies for H-score normalization of preanalytical technical variables with potential utility to immunohistochemical-based biomarker quantitation in therapeutic response diagnostics. Anal Cell Pathol (Amst). 2011,34(3):159-168. doi: 10.3233/ACP2011-014

23. Zhang Z, Xie H, Zuo W, et al. Lysine 2-hydroxyisobutyrylation proteomics reveals protein modification alteration in the actin cytoskeleton pathway of oral squamous cell carcinoma. $J$ Proteomics. 2021,249:104371. doi: 10.1016/j.jprot.2021.104371

24. Zhou Y, Zhou B, Pache L, et al. Metascape provides a biologist-oriented resource for the analysis of systems-level datasets. Nat Commun. 2019,10(1):1523. doi: 10.1038/s41467-019-09234-6

25. Hirashima Y, Kobayashi H, Gotoh J, Terao T. Inter-alpha-trypsin inhibitor is concentrated in the pericellular environment of mouse granulosa cells through hyaluronan-binding. Eur J Obstet Gynecol Reprod Biol. 1997,73(1):79-84.

26. Szarvas D, Gaal B, Matesz C, Racz E. Distribution of the extracellular matrix in the pararubral area of the rat. Neuroscience. 2018,394:177-188. doi: 10.1016/j.neuroscience.2018.10.027

27. Kang JS, Kawakami Y, Bekku Y, Ninomiya Y, Izpisua BJ, Oohashi T. Molecular cloning and developmental expression of a hyaluronan and proteoglycan link protein gene, crtl1/hapln1, in zebrafish. Zoolog Sci. 2008,25(9):912-918. doi: 10.2108/zsj.25.912

28. Xu J, Wang $W$, Ludeman $M$, et al. Chondrogenic differentiation of human mesenchymal stem cells in three-dimensional alginate gels. Tissue Eng Part A. 2008,14(5):667-680. doi: 10.1089/tea.2007.0272

29. Watanabe $H$, Yamada Y. Mice lacking link protein develop dwarfism and craniofacial abnormalities. Nat Genet. 1999,21(2):225-229.

30. Ivanova AV, Goparaju CM, Ivanov SV, et al. Protumorigenic role of HAPLN1 and its IgV domain in malignant pleural mesothelioma. Clin Cancer Res. 2009,15(8):2602-2611. doi: 10.1158/10780432.CCR-08-2755

31. Rockel JS, Bernier SM, Leask A. Egr-1 inhibits the expression of extracellular matrix genes in chondrocytes by TNFalpha-induced MEK/ERK signalling. Arthritis Res Ther. 2009,11(1):R8. doi: $10.1186 /$ ar2595

32. Liu J, Park ES, Curry TJ, Jo M. Periovulatory expression of hyaluronan and proteoglycan link protein 1 (Hapln1) in the rat ovary: Hormonal regulation and potential function. Mol Endocrinol. 2010,24(6):1203-1217. doi: 10.1210/me.2009-0325

33. Sun X, Kaufman PD. Ki-67: More than a proliferation marker. Chromosoma. 2018,127(2):175-186. doi: 10.1007/s00412-018-0659-8 
34. Casimiro MC, Arnold A, Pestell RG. Kinase independent oncogenic cyclin D1. Aging (Albany NY). 2015,7(7):455-456. doi: 10.18632/aging.100773

35. Li Z, Wang C, Prendergast GC, Pestell RG. Cyclin D1 functions in cell migration. Cell Cycle. 2006,5(21):2440-2442. doi: 10.4161/cc.5.21.3428

36. Schwanhausser B, Busse D, Li N, et al. Corrigendum: Global quantification of mammalian gene expression control. Nature. 2013,495(7439):126-127. doi: 10.1038/nature11848

\section{Figures}

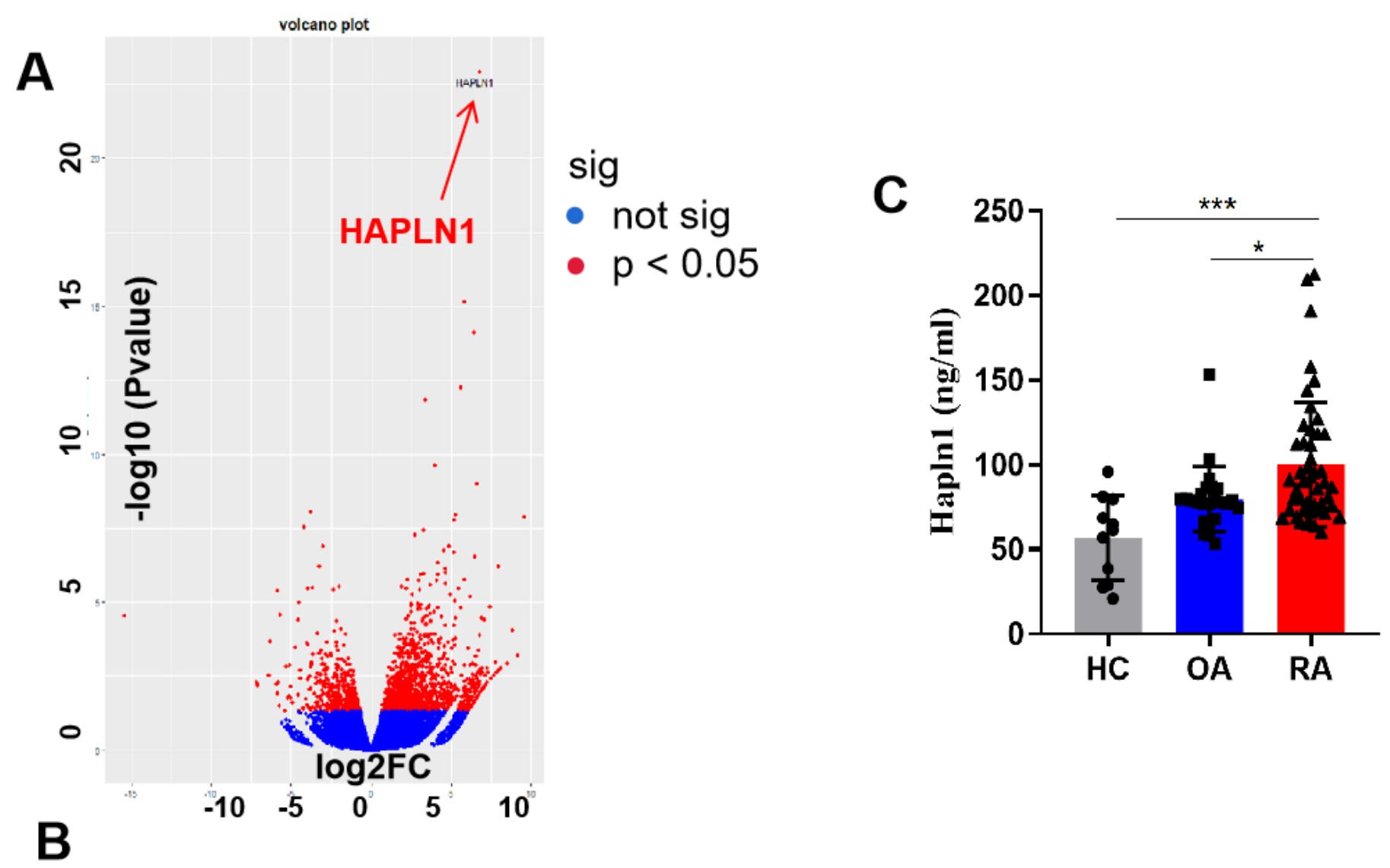

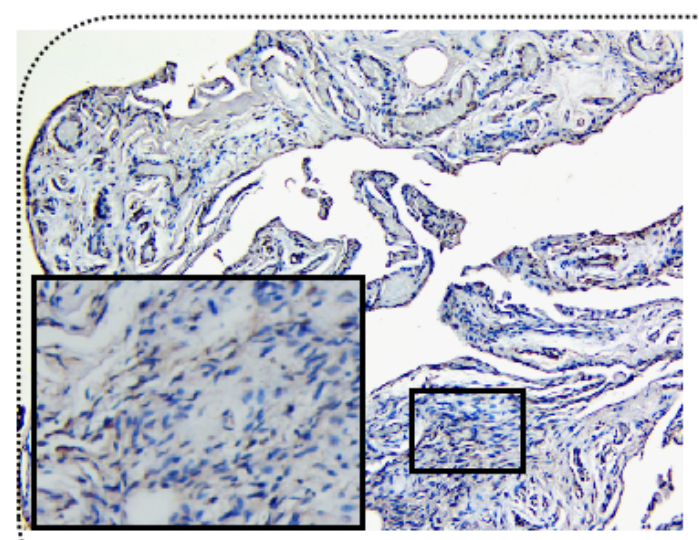

OA

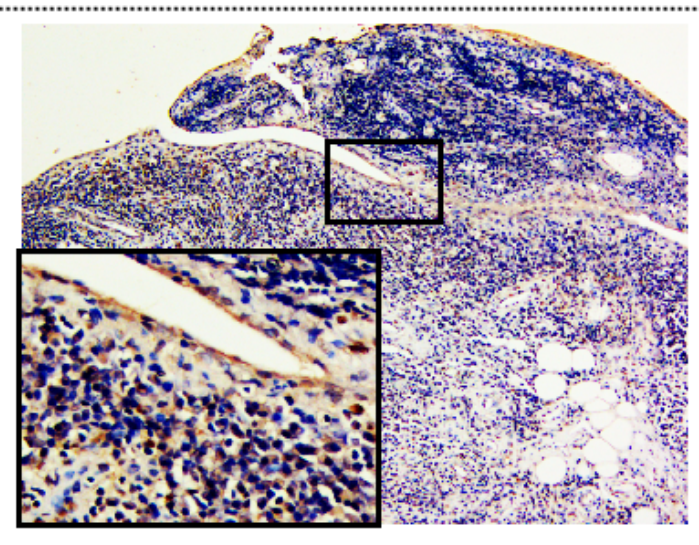

RA

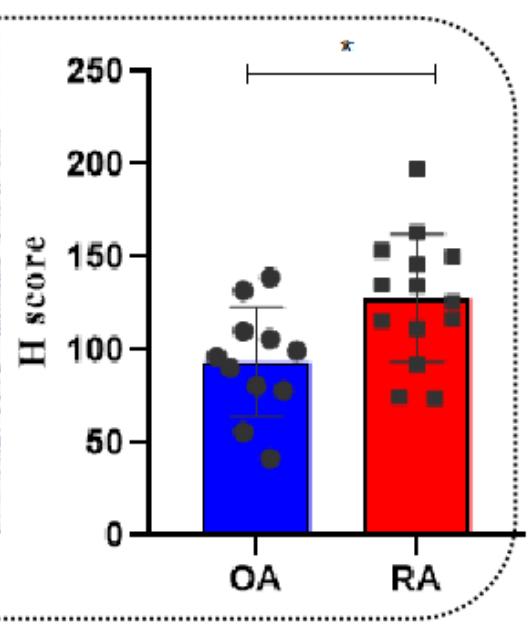

Figure 1 
HAPLN1 is up-regulated in RA patients. (A) Volcano plot showing higher expression of HAPLN1 gene in FLSs from RA than OA patients. (B) Increased HAPLN1 expression in RA than OA synovial membranes (C) Plasma HAPLN1 levels were significantly enhanced in RA patients than OA patients and healthy people. Error bars indicate \pm SD. ${ }^{*} p<0.05,{ }^{* *} p<0.001$.
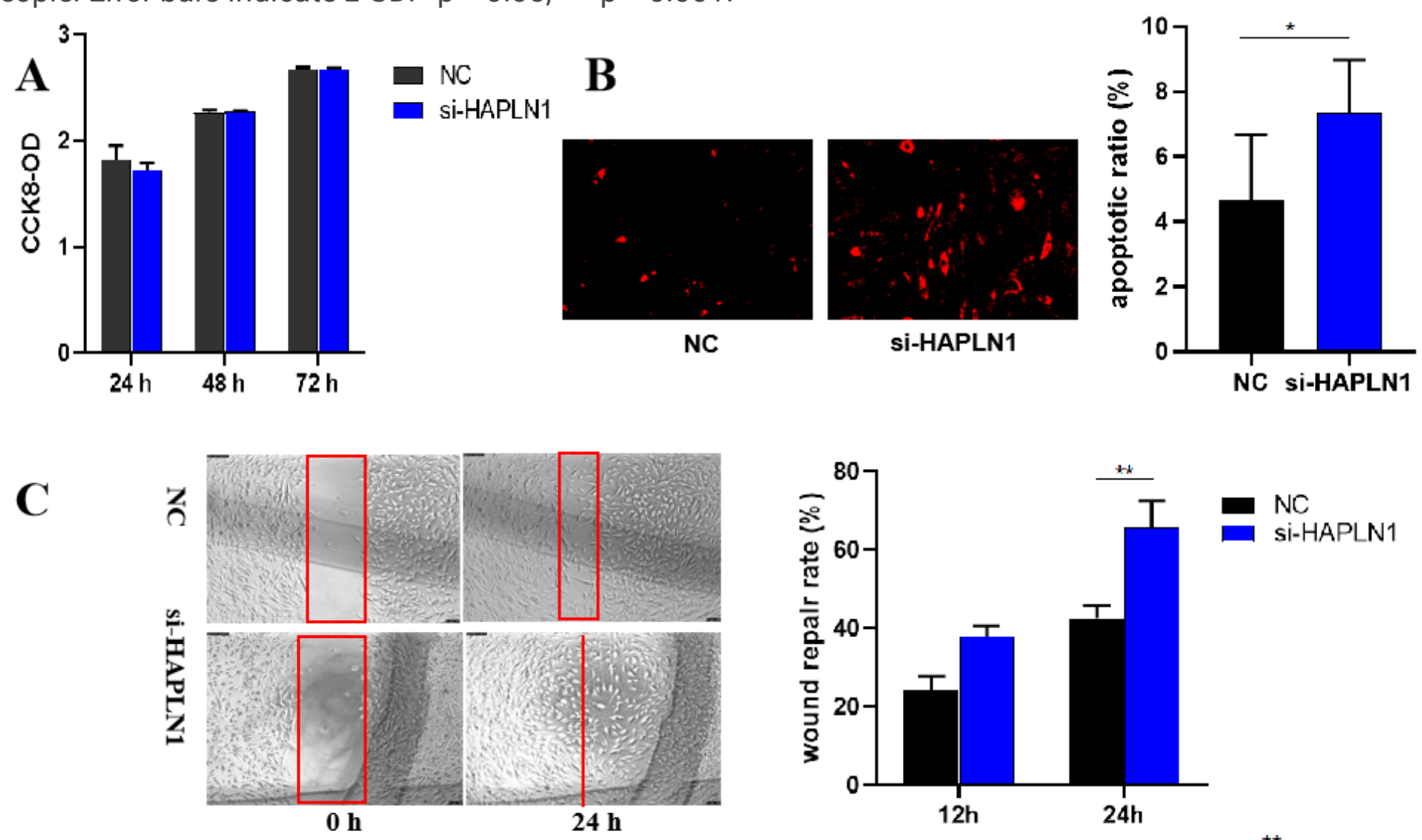

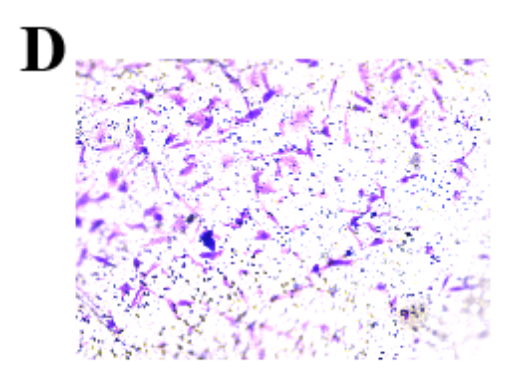

NC

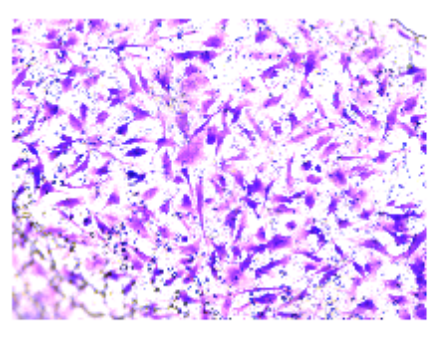

Si-HAPLN1

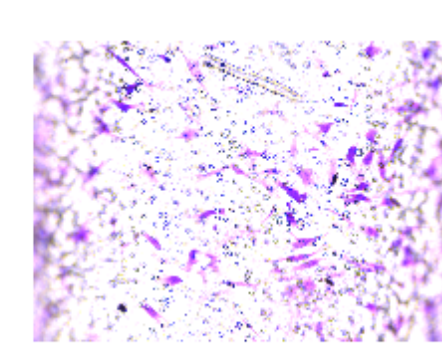

rHAPLN1 $(50 \mathrm{ng} / \mathrm{ml})$
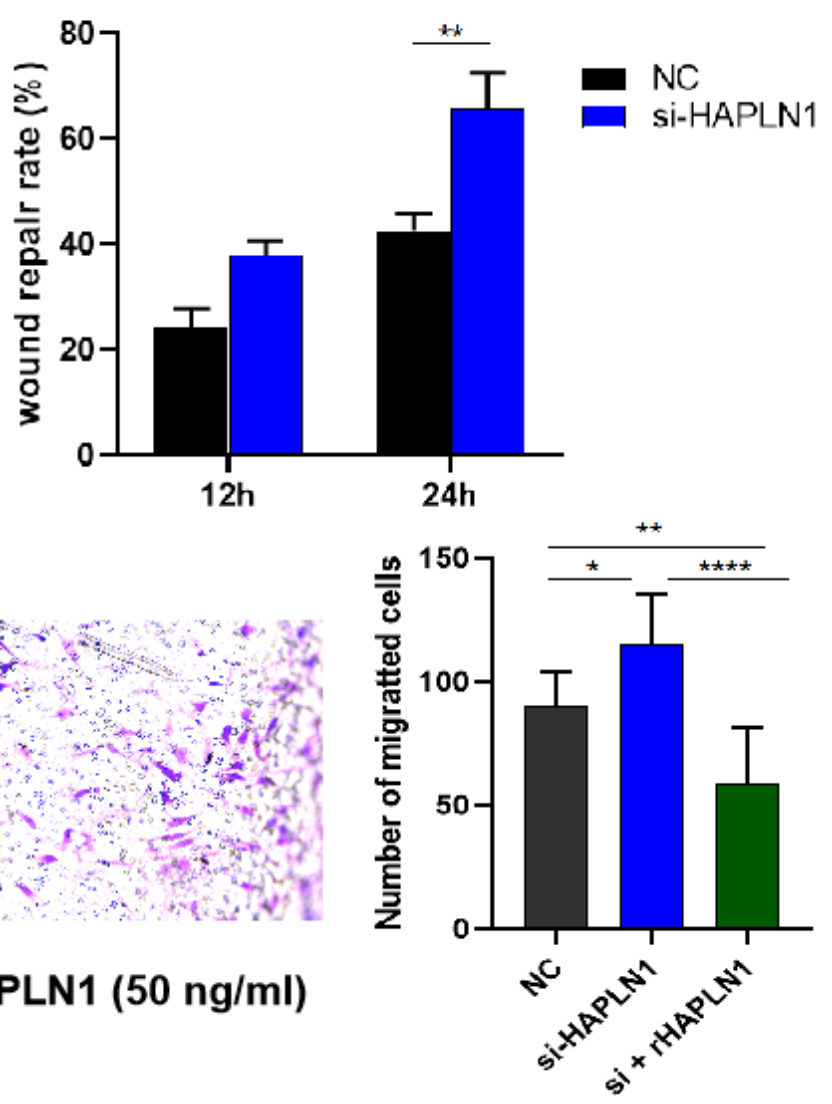

Figure 2

Effect of siHAPLN1 on RA-FLS activity. Transfection of siHAPLN1 in RA-FLSs did not significantly affect their (A) proliferation but significantly increased (B) apoptosis and (C-D) migration ability. Wound healing (C) and transwell (D) assays were used to measure the migration capacity of FLSs. Recombinant HAPLN1 $(50 \mathrm{ng} / \mathrm{ml})$ attenuated the increased migration ability induced by si-HAPLN1 in the transwell assay. Error bars indicate $\pm S D .{ }^{*} p<0.05,{ }^{*} p<0.01,{ }^{\star * \star *} p<0.00001$. 


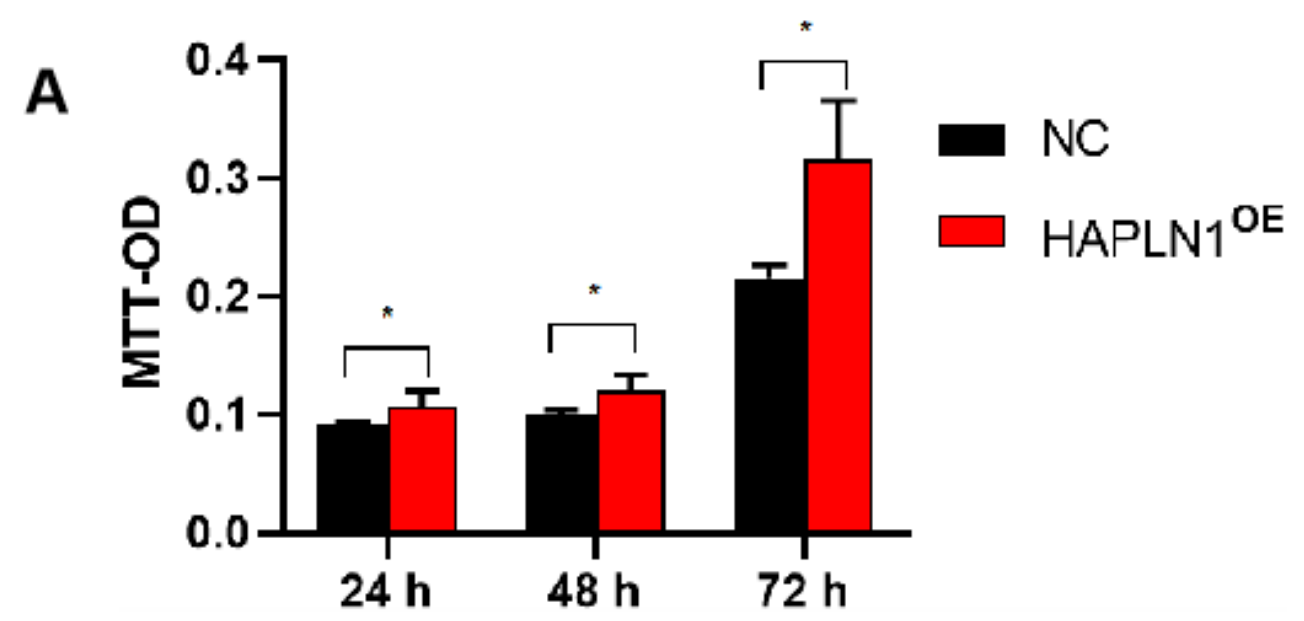

B
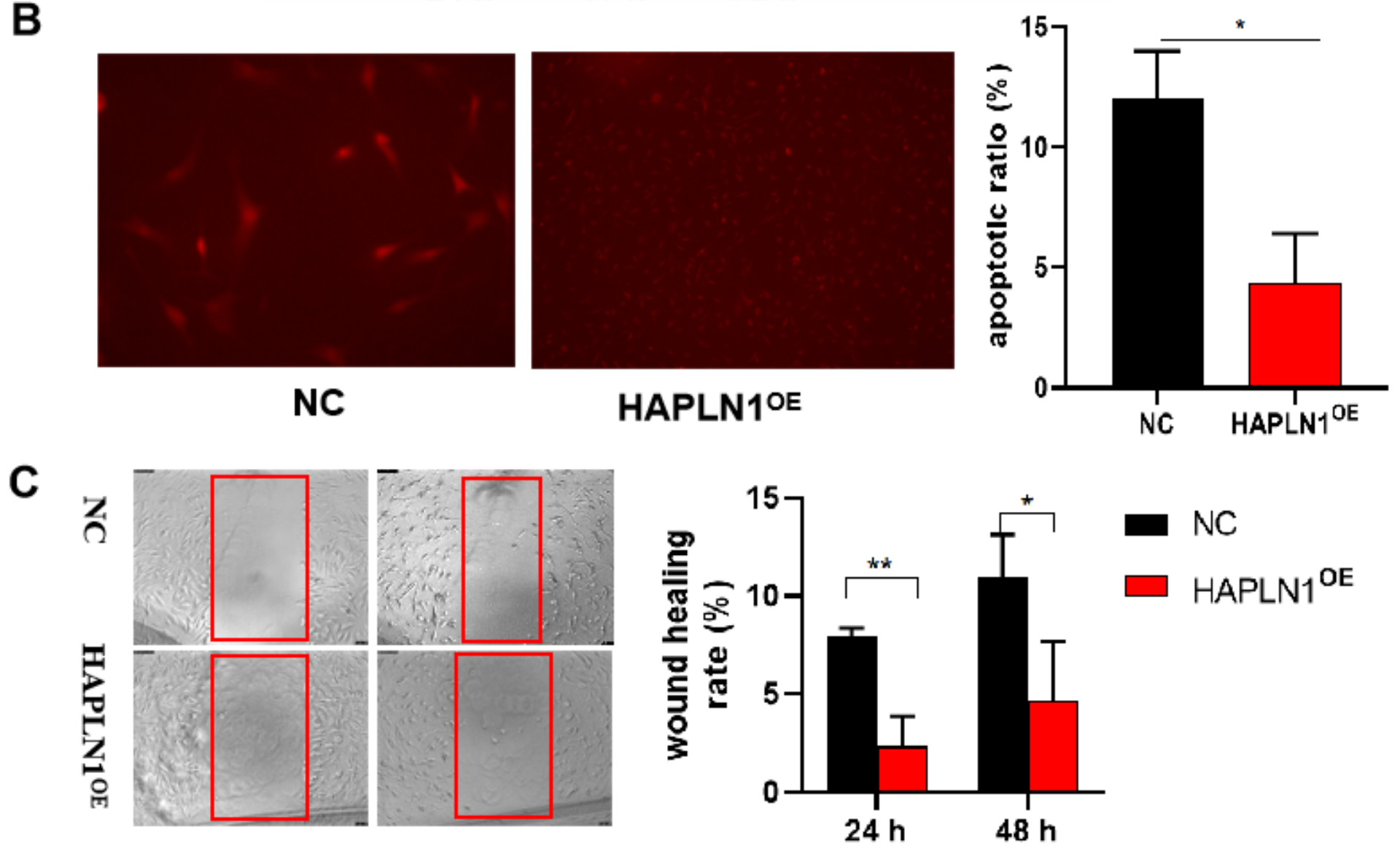

Figure 3

Effect of HAPLN1 over-expression on RA-FLSs activity. Over-expression of HAPLN1 (HAPLN1OE) in RAFLSs has significantly increased their (A) proliferation, but reduced (B) apoptosis and (C) migration. Error bars indicate \pm SD. ${ }^{\star} p<0.05, * \star p<0.01$. 

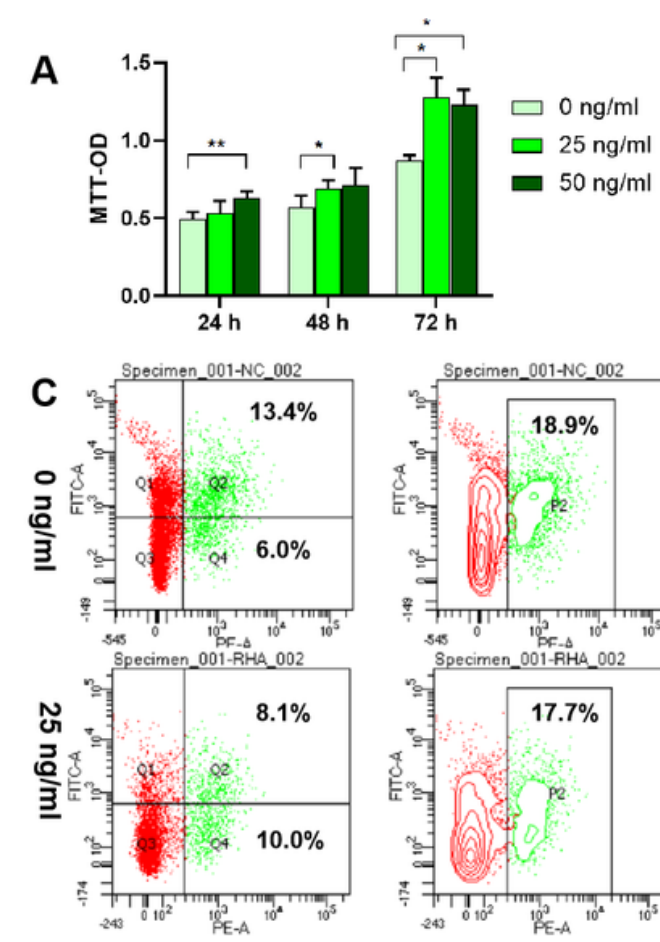

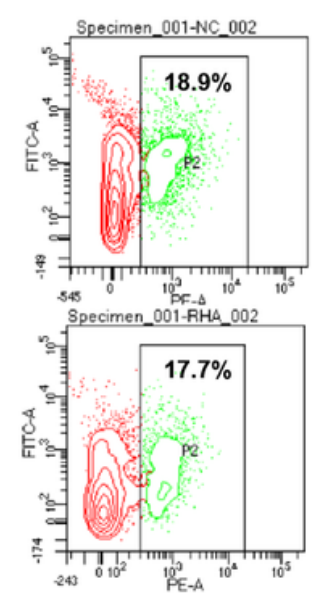

D

B
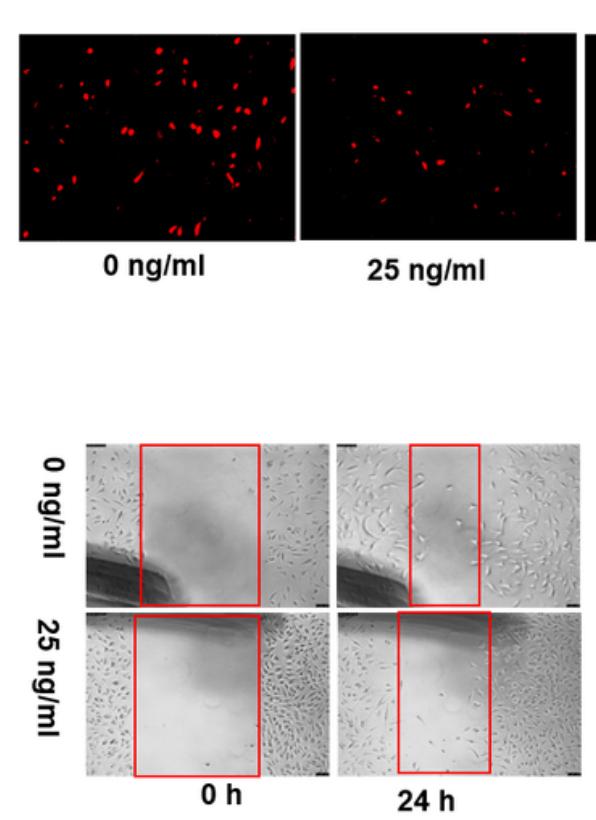

E

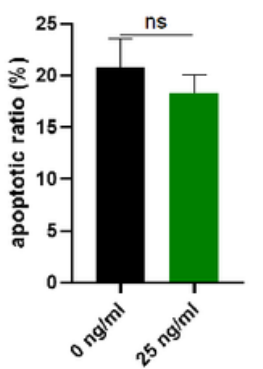

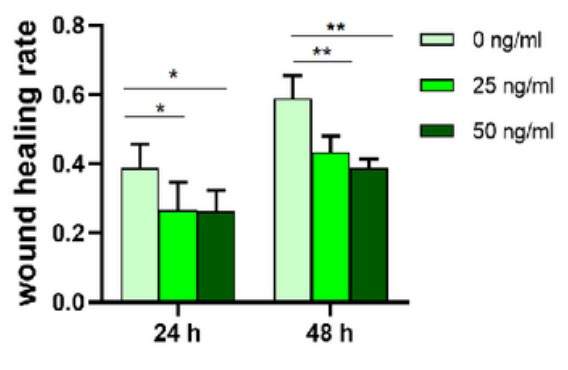
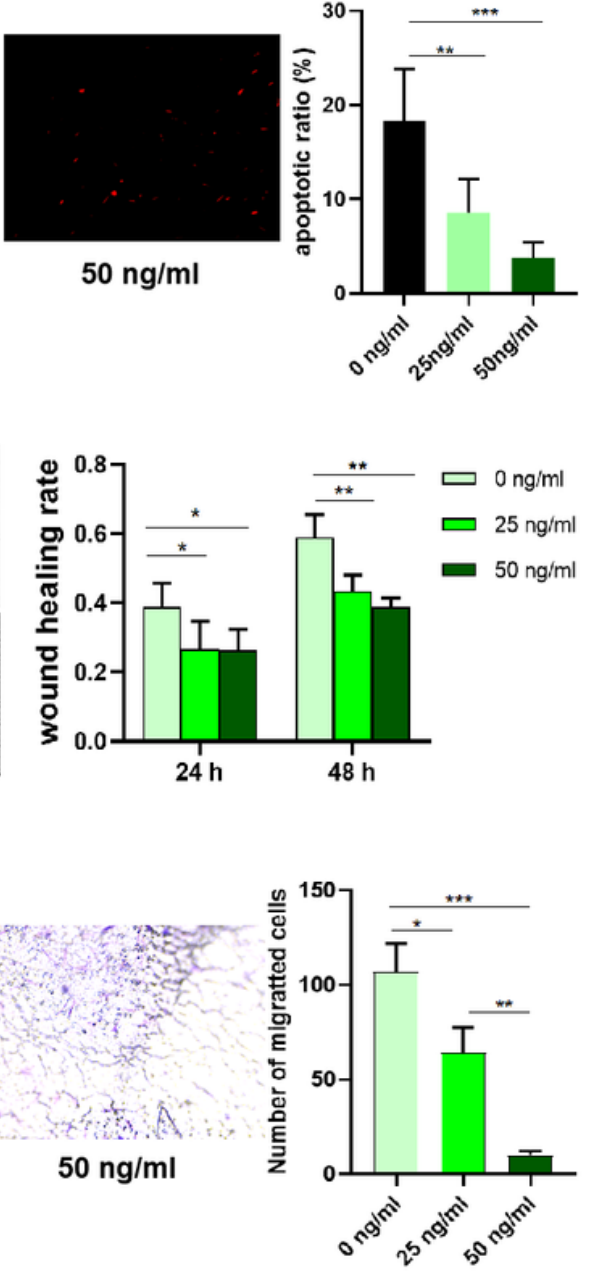

Figure 4

Effect of rHAPLN1 on RA-FLSs activity. (A) Treatment of RA-FLSs with rHAPLN1 has significantly enhanced their $(A)$ proliferation but reduced $(B, C)$ apoptosis, especially during early phase and $(D, E)$ migration. Wound healing (D) and transwell (E) assays were used to evaluate the migration capacity of FLSs. Error bars indicate \pm SD. ns, not significant, ${ }^{\star} p<0.05,{ }^{\star *} p<0.01,{ }^{* \star} \mathrm{p}<0.001$. 


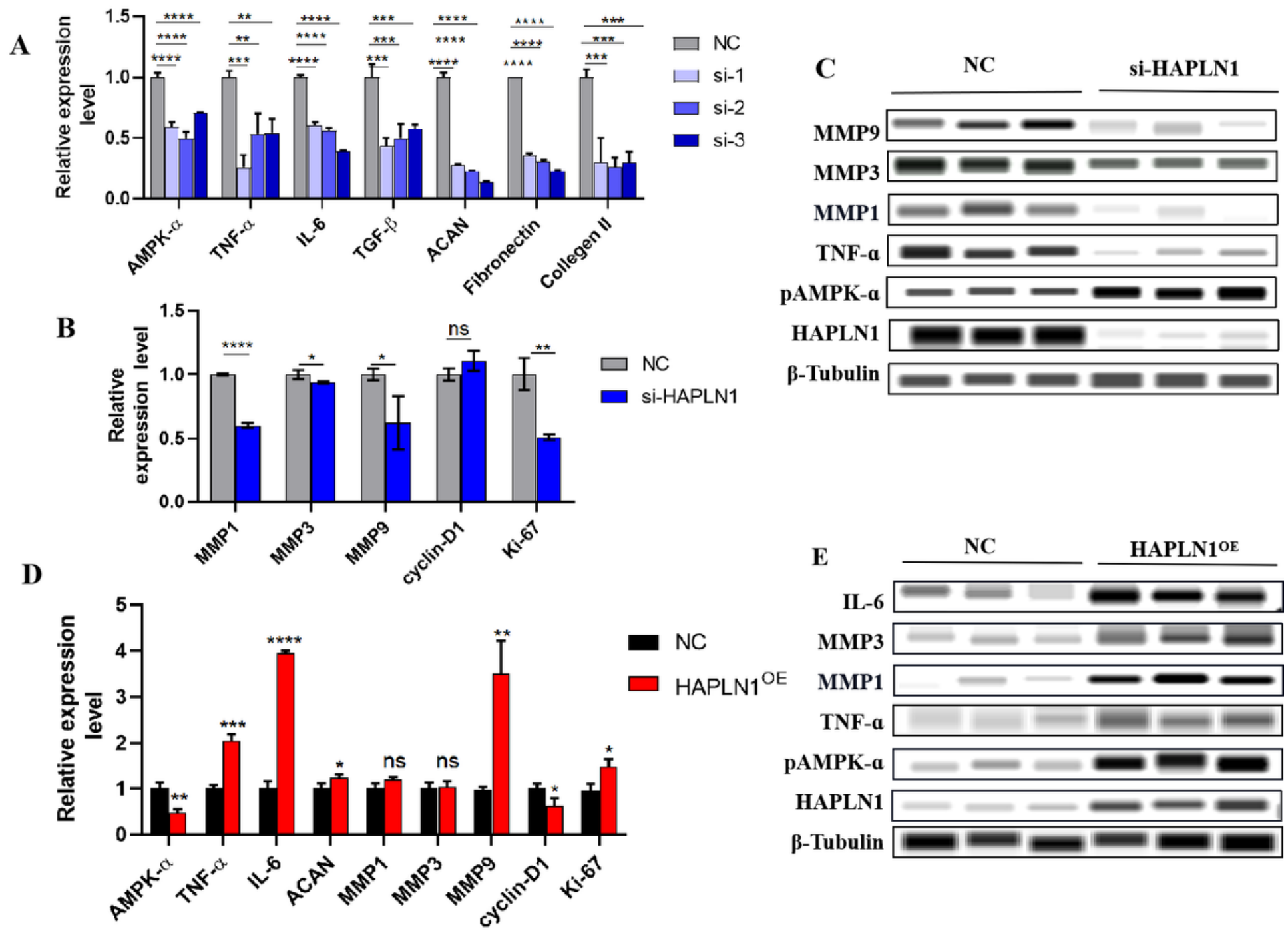

Figure 5

The effect of si-HAPLN1 and HAPLN10E on RA-FLSs derived cytokines and molecules. (A, B) si-HAPLN1 transfection to RA-FLSs inhibited AMPK-a, TNF-a, IL-6, TGF-b, ACAN, Fibronectins, Collagen II, MMP1, MMP3, MMP9 and Ki-67 mRNA expression. (C) At protein level, HAPLN1 has successfully silenced the expression of TNF-a, MMP1, MMP3, and MMP9 except pAMPK-a. (D) After HAPLN1 over-expression in RA-FLSs, TNF-X, IL-6, ACAN, MMP9 and Ki-67 mRNA expressions were up-regulated, while AMPK-囚and cyclin-D1 expressions were down-regulated. (E) Automated WB assay confirmed successful transfection of HAPLN1 over-expression vector into RA-FLSs. Unlike mRNA, the expression of pAMPK- $\$ was up-

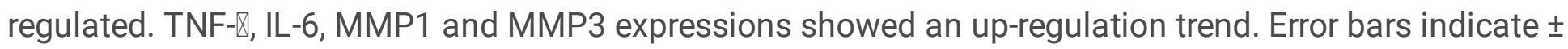
SD. ${ }^{\star} p<0.05,{ }^{\star *} p<0.01,{ }^{\star \star \star} p<0.001,{ }^{\star \star * \star} \mathrm{p}<0.0001, \mathrm{~ns}$, not significant. 
A

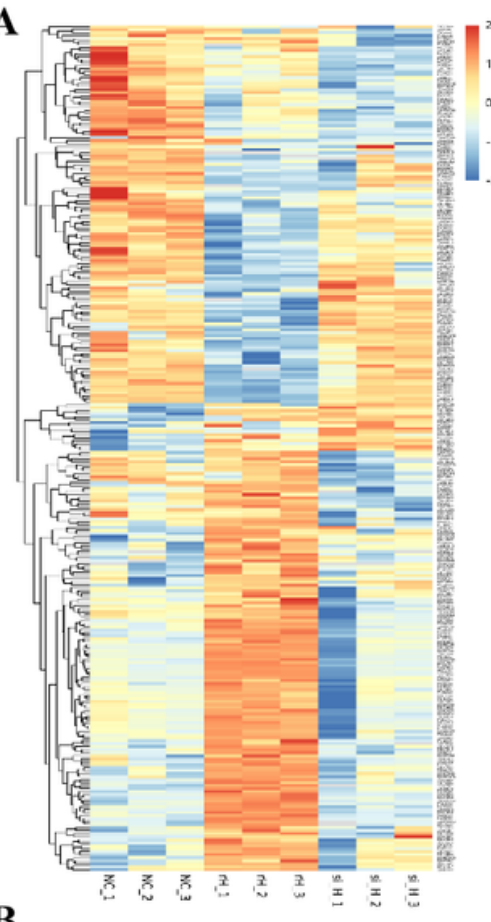

B

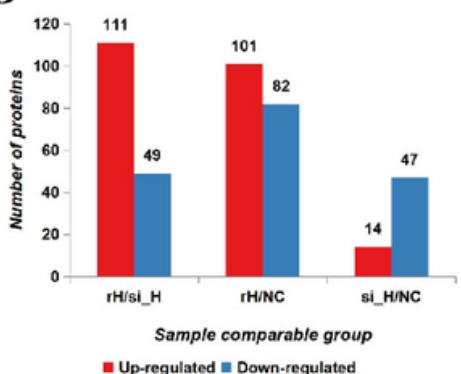

C

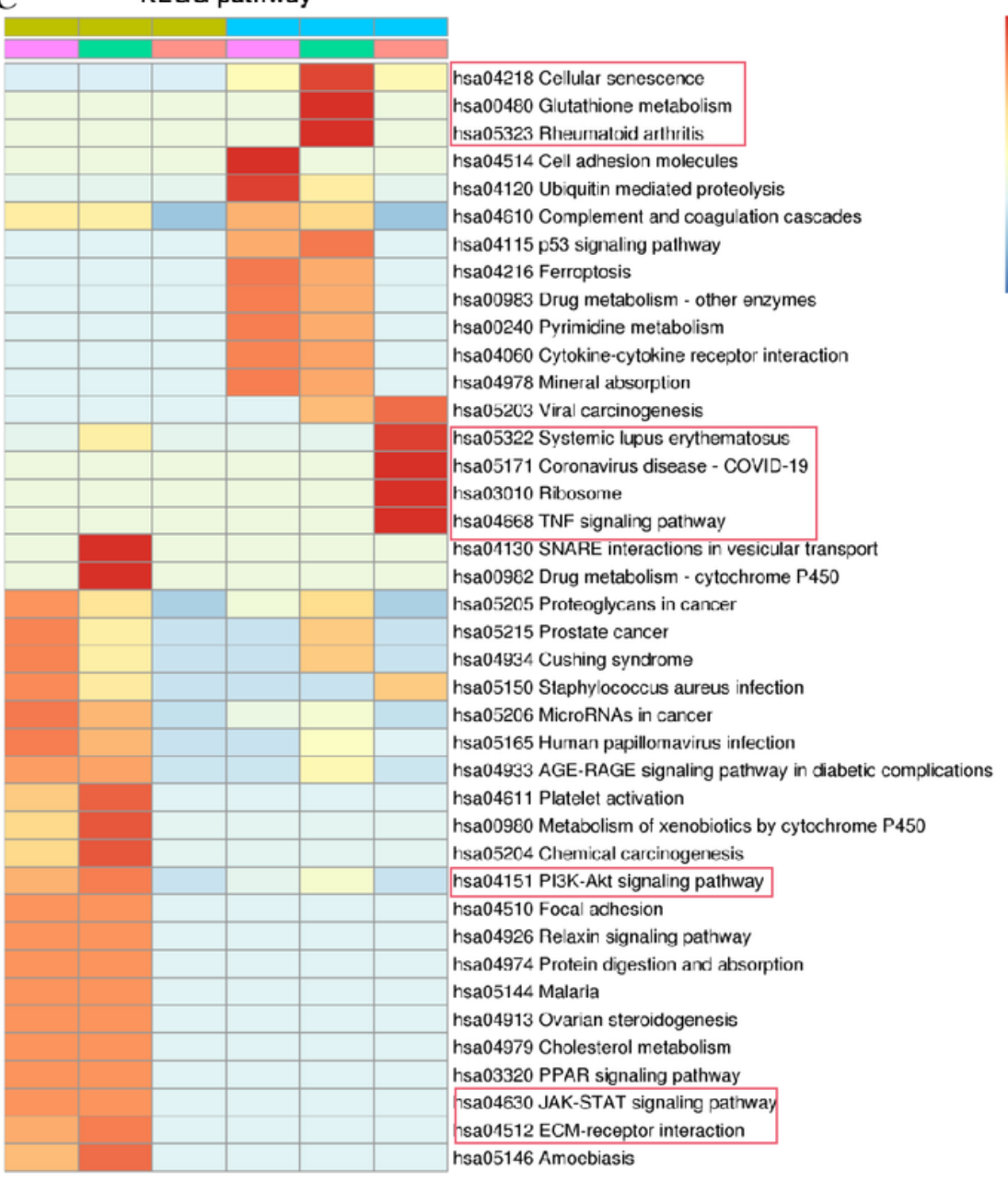

2 Regulated Type Up Down

Sample

0 rHvssi_H rHvsNC si_HvsNC

\section{Figure 6}

Proteomic analysis of si-HAPLN1 and rHAPLN1 treated RA-FLSs. (A \& B) Among the quantifiable proteins, 14 were up-regulated and 47 were down-regulated by si-HAPLN1 transfection. 101 proteins were upregulated, and 82 were down-regulated by rHAPLN1 treatment compared to NC. (C) KEGG enrichment analysis showing the pathways associated with DEGs. rH, recombinant HAPLN1 and si_H, siRNA HAPLN1. 


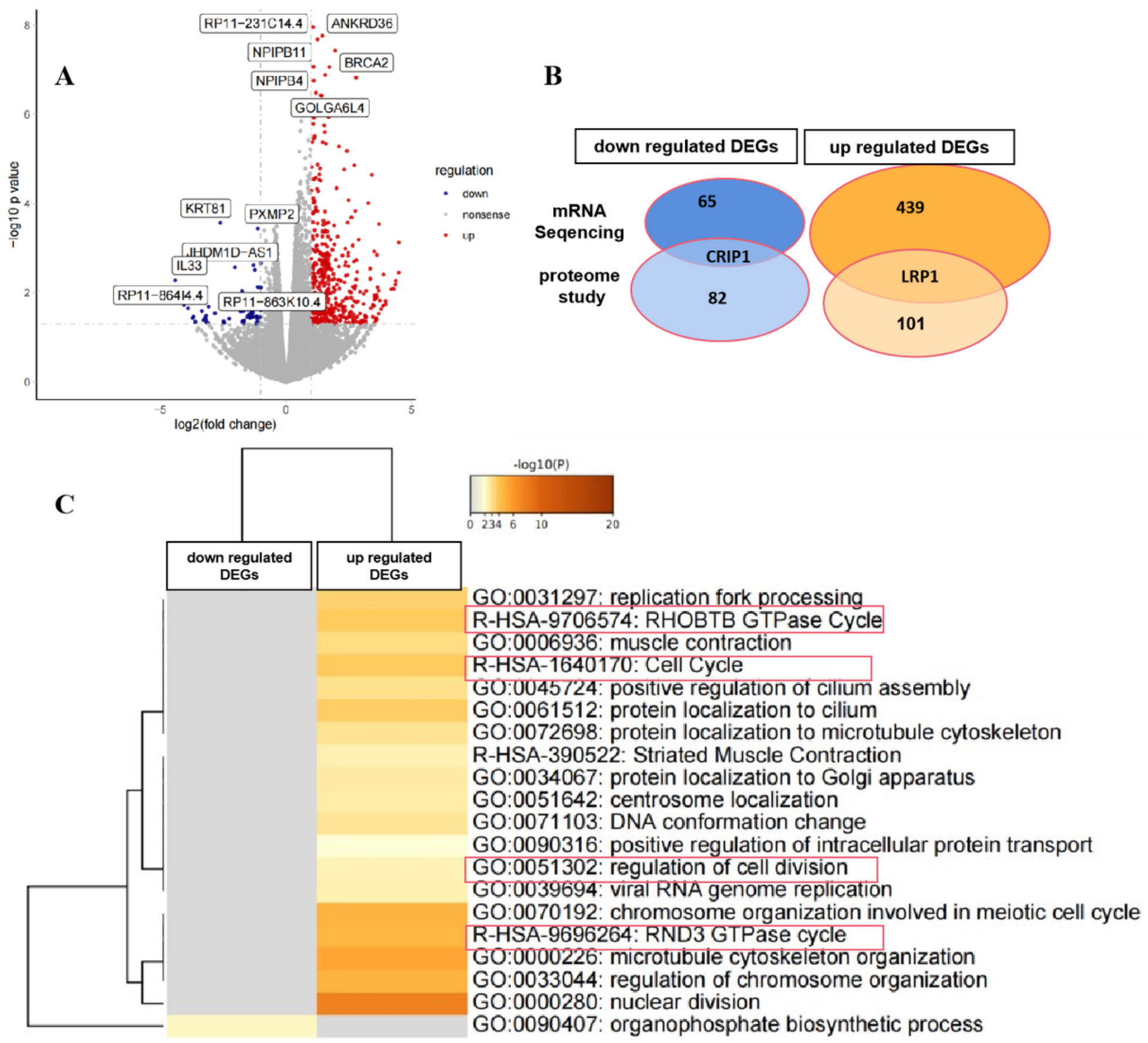

Figure 7

Transcriptome analysis of rHAPLN1 treated RA-FLSs. (A) Among the 504 DEGs detected, 439 were upregulated and 65 were down-regulated. (B) Two DEGs detected (up-regulated LRP1 and down-regulated CRIP1) were in accordance with the proteomic studies. (C) Metascape analysis of DEGs demonstrated an enrichment of various pathways including replication fork processing, GTPase cycle, cell cycle and cell division.

\section{Supplementary Files}

This is a list of supplementary files associated with this preprint. Click to download. 
- Supplementarymaterial2021.11.23Nan.docx 